\title{
Correction to: LiFSI as a functional additive of the fluorinated electrolyte for rechargeable Li-S batteries
}

\author{
Hai Lu ${ }^{1, \star}$ (D), Yan Zhu' ${ }^{1}$, Yan Yuan ${ }^{2, \star}$, Long He ${ }^{1}$, Bin Zheng ${ }^{1}$, Xuezhao Zheng ${ }^{3}$, \\ Changchun $\mathrm{Liu}^{3}$, and Huiling $\mathrm{Du}^{1}$ \\ ${ }^{1}$ School of Materials Science and Engineering, Xi'an University of Science and Technology, Xi'an 710054, China \\ ${ }^{2}$ School of Metallurgical Engineering, Xi'an University of Architecture and Technology, Xi'an 710055, China \\ ${ }^{3}$ College of Safety Science and Engineering, Xi'an University of Science and Technology, Xi'an 710054, China
}

Published online:

19 February 2021

(C) Springer Science+Business

Media, LLC, part of Springer

Nature 2021

Correction to:

Journal of Materials Science: Materials in Electronics

https://doi.org/10.1007/s10854-021-05310-0

In the original version of this article, the title has been published incorrectly. The correct title should read as above "LiFSI as a functional additive of the fluorinated electrolyte for rechargeable Li-S batteries".
This has been corrected by publishing this correction article.

The original article has been updated.

Publisher's Note Springer Nature remains neutral with regard to jurisdictional claims in published maps and institutional affiliations.

The original article can be found online at https:/ / doi.org/10.1007/s10854-021-05310-0.

Address correspondence to E-mail: lhxust@126.com; lingyi21@126.com 